\title{
SIMULADOR DE MINA SUBTERRÂNEA A EVENTOS DISCRETOS PARA APOIO À TOMADA DE DECISÃO
}

Tiago Francioli Souza ' Ivo Eyer Cabral ${ }^{2}$

\section{Resumo}

Objetivo deste trabalho foi demonstrar a utilidade da simulação a eventos discretos, aplicada às operações de mina subterrânea, como ferramenta de apoio à tomada de decisões. Para isso, foi desenvolvido um modelo de simulação de uma mina subterrânea na cidade de Jacobina, utilizando o software Arena. $\bigcirc$ trabalho foi desenvolvido em 03 fases, construção do modelo, validação do modelo e estudos de mudanças estratégicas sobre o sistema analisado. Após a conclusão, comparando os resultados obtidos aos resultados do sistema real, o modelo foi validado. Com o objetivo de demonstrar que a simulação é ferramenta eficaz para o auxílio à tomada de decisão, simulou-se dois novos cenários, onde foram consideradas hipóteses de mudanças estratégicas no sistema. A partir da análise dos novos cenários, foi possível comprovar que a ferramenta simulação a eventos discretos é muito eficaz para suporte à tomada de decisão em mina subterrânea.

Palavras-chave: Pesquisa operacional; Simulação a eventos discretos; Lavra subterrânea.

\section{UNDERGROUND MINE SIMULATOR AT DISCRETE EVENTS TO SUPPORT DECISION MAKING}

\begin{abstract}
The approach of this study was to prove the usefulness of discrete event simulation applied to Underground Mine Operations as a support tool for decision making. The Underground Mine in Jacobina City was simulated in Arena Software. The worked was development in 03 phases: simulation model building, simulation model validation and Studies of strategic changes on the analyzed system. Comparing the results of simulation model to the real system results, the simulation model was validated. With objective to demonstrated that the discrete event simulation is the effecttive tool to support of decision making, two news scenarios were simulated, which were consided hypotheses of strategic changes in the system. From the analyses of news scenarios, it was possible to prove that the simulation tool is very effective to support the decision making analyses about Underground Mine.
\end{abstract}

Keywords: Operational research; Discrete event simulation; Underground mining.

\section{INTRODUÇÃO}

Segundo Freitas [I], mais do que nunca, a simulação computacional tem sido utilizada. $O$ crescimento da utilização dessa ferramenta deve-se, sobretudo, à atual facilidade de uso e sofisticação dos ambientes de desenvolvimento de modelos computacionais, aliadas ao crescente poder de processamento das estações de trabalho.

A simulação tem sido cada vez mais aceita e empregada como uma técnica que permite aos analistas dos mais diversos seguimentos (administradores, engenheiros, biólogos, técnicos em informática, etc.) verificarem ou encaminharem soluções, com a profundidade desejada, aos problemas com os quais lidam diariamente, Freitas [I].

Sakurada e Miyake [2] afirma que a simulação pode ser definida como uma ferramenta de apoio a tomadas de decisão que utiliza modelos para reproduzir um sistema

'Programa de Pós-graduação em Engenharia Mineral, Universidade Federal de Ouro Preto - UFOP, Ouro Preto, MG, Brasil. E-mail: tfsufop@gmail.com

${ }^{2}$ Departamento de Engenharia de Minas, Universidade Federal de Ouro Preto - UFOP, Ouro Preto, MG, Brasil. 
em estudo e resolver problemas cuja solução analítica se mostre inviável.

Outro conceito, apresentado por Steffen e Cassel [3] considera a simulação como uma ferramenta que possibilita expressar uma realidade em um ambiente virtual. Por sua flexibilidade, a Simulação contempla a variabilidade do sistema, gerando resultados dinâmicos, de acordo com os parâmetros estipulados. A sua utilização possibilita uma melhor visualização e entendimento do sistema real.

Ramos [4] cita que a mineração pode ser incluída como sendo um desses sistemas passíveis de serem simulados, por possuir operações complexas, variáveis aleatórias e por ser necessária a avaliação de estratégias para estas operações.

O Software Arena, utilizado para construção do Simulador em questão, é um ambiente gráfico integrado de simulação, que contém todos os recursos para modelagem, animação, análise estatística e análise de resultados.

Segundo Prado [5], tal como a maioria dos softwares de simulação, o Arena visualiza o sistema a ser modelado como constituído de um conjunto de estações de trabalho que prestarão serviços a entidades que se movem através do sistema. O movimento pode ser feito pela própria entidade ou por transportadores ou correias.

O sistema real simulado via software Arena, foi uma mina subterrânea situada na cidade de Jacobina, estado da Bahia, que utiliza o método de lavra sublevel open stoping (desmonte em subníveis), com capacidade nominal média de $3.500 \mathrm{t} /$ dia de minério para alimentação da planta metalúrgica, perfazendo um total aproximado de $100.000 \mathrm{t} / \mathrm{mês}$ em regime de 30 dias/mês e aproximadamente I,2 milhão de toneladas/ano.

Visando garantir o avanço do desenvolvimento e lavra do minério, a empresa possui uma frota de equipamentos de perfuração, carregadeiras, caminhões e equipamentos de apoio.

Logo, os equipamentos da mina se revezam nas frentes, dependendo do estado em que cada frente se encontra. Enquanto uma frente está sendo perfurada, outra já foi detonada, outra está em carregamento. A sequência das atividades em qualquer frente é sempre perfurar, detonar, carregar e transportar. Logo, cria-se um ciclo de atividades, onde sempre se tem minério e estéril disponíveis para carregamento e transporte.

Neste trabalho procurou-se demonstrar, através do software Arena, que a simulação computacional, é uma ferramenta útil para análises estratégicas das atividades operacionais de extração de minério em mina subterrânea, a partir do desenvolvimento de um simulador dessa mina em Jacobina, e utilização do mesmo para análises estratégicas de mudança no sistema real.

\section{MATERIAIS E METÓDOS}

Diversos termos técnicos são utilizados em projetos de simulação. As entidades são a parte dinâmica do sistema a ser analisado, ou seja, o que moverá entre as estações de trabalho, que são os pontos onde ocorrerá alguma ação. Os atributos são as características próprias das entidades, isto é, aquelas que a definem totalmente. Um recurso, segundo Freitas [I], é considerado uma entidade estática que fornece serviços às entidades dinâmicas, em uma estação de trabalho. Já as Variáveis de estado determinam o estado de um sistema, sendo o conjunto de informações necessárias à compreensão do que está ocorrendo no sistema.

O sistema simulado foi o processo de extração de minério e estéril de uma mina subterrânea em Jacobina, estado da Bahia, e o processo de transporte de minério do Pátio de estocagem à planta de beneficiamento.

O maior prazo para o programa de produção dessa mina é o plano de vida da mina, que se baseia na reserva mineral e no modelo de aproveitamento da mesma. A partir daí todo ano este plano é reprogramado gerando o Plano Anual de Produção.

Todo mês o departamento de planejamento de mina faz a programação com objetivo de apresentar as metas do mês de forma detalhada, ou seja, as frentes de desenvolvimento e os blocos de lavra que serão extraídos. A programação mensal pode ser igual ao apresentado no Plano Anual de Produção ou diferente dependendo da situação atual da mina.

O modelo desenvolvido simulou a programação de um mês especifico que considerou 25 (vinte e cinco) frentes de desenvolvimento exploratório, onde serão desenvolvidos uma média de 32 (trinta e dois) metros por frente, 10 (dez) frentes de desenvolvimento produtivo e 8 (oito) blocos de lavra. A partir deste plano, a Gerência de Operação das Minas deve fazer a programação das atividades dos equipamentos disponíveis para cumprimento da meta.

A principal característica que se procurou desenvolver no modelo foi a flexibilidade, visando facilitar a adaptação do simulador aos cenários a serem analisados. Para isso foi desenvolvido uma interface do modelo com um arquivo de entrada de dados desenvolvido no software Microsoft Excel. Desta forma, todas as características do modelo, tais como frentes a serem trabalhadas, principais distâncias, número de equipamentos e respectivas disponibilidades, deverão ser inseridas via Excel.

Neste contexto, usuários que não tenham conhecimento de programação na linguagem do software Arena poderão utilizar o modelo para análises, sem dificuldades, fazendo alterações nas características do sistema via arquivo Excel e rodando o modelo sem complicações.

Para construção do simulador foram definidos os componentes do modelo, apresentados a seguir.

\section{I Entidades e Atributos}

No processo de extração da mina, o principal objetivo é alimentar a planta de beneficiamento com o minério extraído. Considera-se também, que no desenvolvimento exploratório, ocorre a movimentação de estéril. Como o 
minério e o estéril são transportados por caminhões, este é uma das entidades do sistema. Logo os caminhões, entidades do modelo de simulação, são atendidos pelas estações de trabalho, carga, descarga ou manutenção.

Foram criadas também entidades para cada frente considerada no plano mensal simulado, visando representar as atividades perfuração, desmonte e suporte de mina. O objetivo principal destas entidades foi informar através de variáveis, as frentes que estarão com minério ou estéril disponíveis para carregamento, visando determinar o fluxo das entidades caminhões no ambiente de simulação. Logo os dois ambientes, atividades nas frentes de extração e o ciclo dos caminhões interagem entre si através das variáveis materiais (minério e estéril) disponíveis para transporte. A Figura I ilustra graficamente o modelo:

De acordo com a Figura I, o simulador recebe via Excel todas as informações do período a ser simulado, através do arquivo de entrada de dados (Plano de Produção, equipamentos, disponibilidades, distâncias, velocidades, etc). Após ler as informações, o simulador roda o modelo, através da interface das atividades das frentes com os ciclos dos caminhões, fornecendo os resultados para análise.

\subsection{Estações de Trabalho}

As estações de trabalho são os itens do simulador onde as entidades caminhões ou frentes de lavra ou desenvolvimento ocupam alguma parte do seu tempo desenvolvendo uma atividade. As principais estações de trabalho do sistema são os locais de carga e descarga de minério ou estéril para os caminhões, e as atividades de perfuração, desmonte e preparação da frente, para as entidades das frentes. Logo os caminhões, trabalham em um ciclo constante de carga de minério ou estéril e descarga destes nos seus respectivos destinos. As entidades que representam as atividades das frentes são atendidas em estações de trabalho das atividades de perfuração, desmonte e preparação de acordo com a disponibilidade e quantidade dos equipamentos de perfuração, recursos de carregamento de explosivos, etc.

\subsection{Recursos}

Nas estações de trabalhos os caminhões são atendidos por recursos, as carregadeiras, no caso do carregamento. Logo se as carregadeiras não estiverem disponíveis nas estações de trabalho, por motivo de manutenção ou por estarem em outra frente, os caminhões terão que esperar até que estas estejam disponíveis para continuarem os ciclos de carga e descarga, ou dirigirem-se para outra frente de carga. O mesmo procedimento vale para os outros equipamentos, tais como, equipamentos de perfuração, equipamento de carregamento de explosivos, onde cada frente espera a disponibilidade do equipamento para realizar a atividade específica.

\subsection{Variáveis do Modelo}

No modelo de simulação construído foram consideradas ao todo I56 (cento e cinquenta e seis) variáveis para caracterização dos acontecimentos do sistema real, que representam desde a disponibilidade de equipamentos aos teores do minério em cada bloco de lavra.

\subsection{Modelo Lógico do Simulador}

Para que o simulador represente o sistema real, as entidades precisam mover-se pelo sistema. Como se têm várias opções de rotas para movimentação dos caminhões que entram na mina para retirada de minério ou estéril, diversos artifícios foram utilizados para representação destas atividades. As premissas adotadas foram:

- No modelo, as entidades caminhões para lavra e desenvolvimento foram criadas separadas;

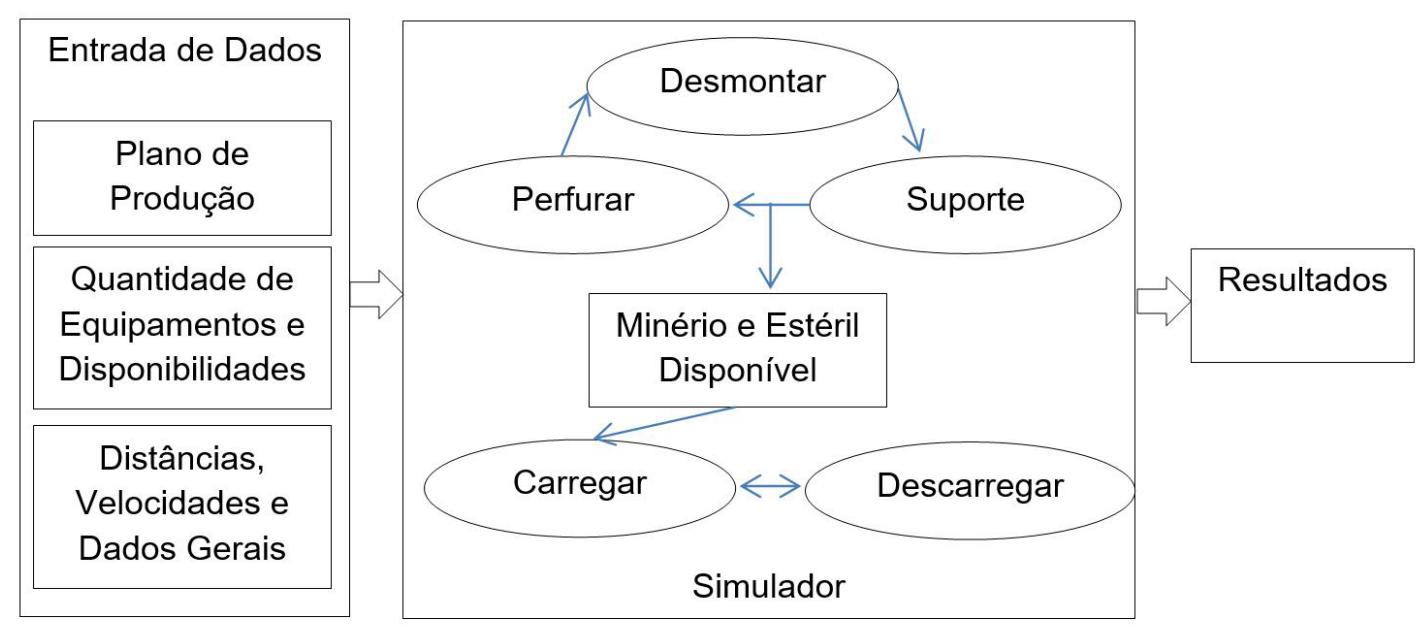

Figura I. Fluxo de informações do simulador.

Tecnol. Metal. Mater. Miner., São Paulo, 
- Tanto no desenvolvimento quanto na lavra, o caminhão precisa decidir em que frente irá carregar. Uma resposta óbvia é que o caminhão irá para frente onde se tem minério e carregadeira disponível. Esta decisão é feita no modelo através de um teste que verifica se a frente prioritária está disponível, ou seja, se tem material a ser carregado, valor indicado pela variável quantidade de material na frente, definido pela entidade de cada frente. Se a condição ter material disponível para o carregamento não for satisfeita, testa-se a segunda opção de frente e assim sucessivamente. Além da condição ter material na frente, testa-se também se há carregadeira disponível para realizar o carregamento. Ressalta-se que se algum caminhão já foi enviado a uma frente, o modelo testa se tem outra frente que está disponível e com carregadeira, caso contrário, espera-se na fila, até uma frente apresentar todas as condições necessários para o carregamento. A variável material disponível na frente é determinada pelas atividades na frente: Perfurar, desmontar, etc., logo só é disponibilizado material para carregamento após o ciclo de atividades na frente estiver completo;

- Quando inicia o processo de carregamento em uma frente, ou seja, é satisfeita a condição da variável material na frente ser maior do que zero, ocupa-se o recurso carregadeira até a variável material na frente atingir o valor zero, ou seja, até que todo o material seja transportado;

- As variáveis material disponível na frente e material planejado para a frente vão diminuindo à medida que uma entidade caminhão passa na estação de trabalho;

- Quando a variável material na frente zerar, ou seja, o material na frente acabar, libera-se o recurso carregadeira e é emitido um sinal para a entidade da respectiva frente para início de um novo ciclo de perfuração, desmonte e preparo, considerando a disponibilidade dos recursos equipamentos de perfuração, equipamentos de carregamento de explosivos para desmonte, etc.;

- Depois de carregado em uma das frentes, a entidade caminhão terá que se deslocar até a estação de trabalho
Pátio de Estocagem em caso de minério, e para o respectivo destino em caso de estéril. Chegando ao destino o modelo somará a capacidade de um caminhão à variável total de minério transportado e o mesmo valor à variável estoque no pátio;

- Para as entidades caminhões Scanias, que trabalham no transporte de minério entre o pátio de estocagem e a planta de beneficiamento, o modelo testará se tem minério no pátio. Caso positivo, a entidade passa pela estação de trabalho carregamento, e diminui-se a capacidade do caminhão da variável total de estoque no pátio. A partir daí a entidade caminhão vai para a estação de trabalho britador, descarrega e volta, aumentando em uma capacidade do caminhão a variável alimentação na planta.

\section{RESULTADOS}

Depois da construção do simulador, foram coletados dados referentes à Programação Mensal de produção de um mês aleatório para utilização do mesmo como protótipo para validação do modelo. Com as informações referentes a este mês específico, o modelo foi rodado e apresentou um resultado satisfatório em relação à realidade. $A$ Tabela $I$ mostra um comparativo dos resultados do simulador com o resultado real obtido no mês aleatório selecionado.

Uma consideração importante em relação aos resultados obtidos, é que o modelo considera que a operação irá seguir o plano de produção em termos de quantidades e prioridades de trabalho nas frentes da mina. Logo o simulador é uma ferramenta de apoio ao planejamento que prevê o resultado das atividades caso este plano seja seguido.

Desta forma, a variável estéril transportado que apresentou resultados reais diferentes do simulador consiste em não seguimento do Plano Mensal de Produção pela Operação durante a realização das atividades. Considera-se que $o$ simulador seguiu as prioridades de frentes de desenvolvimento passadas na Programação Mensal de Produção, o que não aconteceu na realidade, conforme confirmado em campo.

Neste contexto, com exceção da variável estéril é possível observar que todas as outras variáveis tiveram

Tabela I. Resultados obtidos com o Simulador versus resultados do sistema real

\begin{tabular}{|c|c|c|c|c|c|}
\hline \multirow{2}{*}{ Variáveis } & \multirow{2}{*}{ Und } & \multicolumn{3}{|c|}{ Resultados Simulador } & \multirow{2}{*}{ Resultado Real } \\
\hline & & Mínimo & Máximo & Média & \\
\hline Minério Extraído & ton & 87.590 & 95.436 & 91.513 & 95.379 \\
\hline Estéril Extraído & ton & 18.303 & 20.179 & 19.241 & 10.644 \\
\hline Minério transportado ao britador & ton & 93.270 & 101.398 & 97.334 & 96.162 \\
\hline Desenvolvimento da Mina & $M$ & 394 & 410 & 402 & 396 \\
\hline Teor médio minério extraído & $\mathrm{g} /$ ton & $\mathrm{I}, 7$ & 2,11 & 1,905 & 1,98 \\
\hline
\end{tabular}


Tabela 2. Resultados estimados a partir da mudança do regime de turno

\begin{tabular}{|c|c|c|c|c|c|}
\hline \multirow{2}{*}{ Variáveis } & \multirow{2}{*}{ Und } & \multicolumn{3}{|c|}{ Resultado Simulador } & \multirow{2}{*}{ Ganho \% } \\
\hline & & Mínimo & Máximo & Média & \\
\hline Minério Extraído & ton & 100.099 & 105.934 & 103.016 & 12.6 \\
\hline Estéril Extraído & ton & 22.548 & 22.950 & 22.749 & 18.2 \\
\hline Minério transportado ao britador & ton & 105.700 & 111.339 & 108.519 & 11.5 \\
\hline Desenvolvimento da Mina & $M$ & 439 & 452 & 445 & 10.8 \\
\hline Teor médio minério extraído & $\mathrm{g} /$ ton & $\mathrm{I}, 56$ & 2,12 & $\mathrm{I}, 84$ & $-3,4$ \\
\hline
\end{tabular}

Tabela 3. Resultados estimados para estudo da capacidade da mina

\begin{tabular}{lccccc}
\hline \multicolumn{1}{c}{ Variáveis } & \multirow{2}{*}{ Und } & \multicolumn{3}{c}{ Resultado Simulador } & \multirow{2}{*}{ Ganho \% } \\
\cline { 3 - 5 } & & Mínimo & Máximo & Média & \\
\hline Minério Extraído & ton & 126.042 & 130.213 & 128.127 & 40 \\
Estéril Extraído & ton & $22.28 \mid$ & 26.299 & 24.290 & 26 \\
Minério + Estéril & ton & 148.323 & 156.512 & 152417 & 38 \\
Minério transportado ao britador & ton & 131.858 & 136.039 & 133.948 & 38 \\
Desenvolvimento da Mina & M & $7 \mid 4$ & 735 & 724 & 80 \\
Teor médio minério extraído & g/ton & 1,56 & 2,12 & 1,84 & $-3,4$ \\
\hline
\end{tabular}

resultados reais dentro dos intervalos mínimos e máximos, validando assim o Simulador.

A análise de cenários futuros foi baseada em projetos em fase de desenvolvimento na empresa.

Foram analisados dois cenários:

- Troca do sistema de turnos de trabalho de 06 para 08 horas, com aumento efetivo de 01 hora efetiva de trabalho.

- Análise da capacidade de produção da mina visando avaliar seu desempenho para o plano de 8.000 toneladas/dia. Ressalta-se que para análise da capacidade da estrutura atual, foi considerado que a mina possui minério em quantidade ilimitada para extração, dessa forma a quantidade de minério não seria gargalo, logo poderia ser medida a capacidade de extração da estrutura de equipamentos existente.

O objetivo destas análises foi levantar o efeito sob as atividades da empresa em termos de produção, ou seja, porcentagem de aumento ou perda de produção, com a adoção das alternativas citadas.

A partir das mudanças efetuadas no arquivo Entrada de Dados, foi rodado o modelo e os resultados obtidos são apresentados nas Tabelas 2 e 3.

Após os resultados obtidos via simulação é possível concluir que a empresa teria ganhado em média $12,6 \%$ na extração de minério e aproximadamente $10,8 \%$ em desenvolvimento total, caso tivesse adotado o novo regime de turno e considerando o programa de produção do mês aleatório selecionado.

A partir dos resultados acima, é possível concluir que caso seja fornecido um Plano de Produção ilimitado para um mês, com a estrutura de equipamentos atual e considerando o turno de 08 horas, a empresa teria um ganho médio de aproximadamente $38 \%$ em tonelagem movimentada (estéril + minério) e $80 \%$ em desenvolvimento. Concluindo que a atual limitação da mina é a reserva de material disponível para trabalho e não os equipamentos.

\section{CONCLUSÕES}

A partir do término do projeto, conclui-se que a simulação realmente é uma ferramenta eficaz para suportar decisões relacionadas à mineração subterrânea. $\mathrm{A}$ análise dos cenários futuros também foi satisfatória considerando as informações que se teve acesso.

A partir dos relatórios do software Arena, é possível levantar características do sistema analisado, que muitas vezes não são vistas na rotina real do sistema, que podem vir a ser utilizadas em atividades de tomada de decisões na empresa.

Outro ponto importante será a utilização do software Arena na validação dos Planos de Produção desenvolvidos pelo Planejamento de Mina. A partir do simulador será possível estudar se a atual estrutura é suficiente para conclusão do Plano de Produção proposto.

Neste contexto, concluiu-se que o Arena é uma ferramenta muito eficaz na análise da situação atual do sistema analisado, análises essas que podem ser utilizadas como ferramenta de apoio nas atividades gerenciais, e também na análise de possíveis cenários futuros, que consideram mudanças estratégicas no sistema real. 


\section{REFERÊNCIAS}

I Freitas PJ. Fo. Introdução a modelagem e simulação de sistemas com aplicações em Arena. 2. ed. Florianópolis: Visual Books; 2008.

2 Sakurada N, Miyake I. Estudo comparativo de softwares de simulação de eventos discretos aplicados na modelagem de um exemplo de loja de serviços. In: Associação Brasileira de Engenharia de Produção. XXIII ENEGEP - Encontro Nacional de Engenharia de Produção; 2003; Ouro Preto, Brasil. Porto Alegre: ABEPRO; 2003.

3 Steffen DA, Cassel RA. Relatório final da simulação do abastecimento da TNT no condomínio industrial da General Motors (GM). Canoas: Centro Universitário La Salle; 2005.

4 Ramos AN. No. Desenvolvimento de um template no programa Arena para a simulação das operações de carregamento e transporte em minas a céu aberto (dissertação de mestrado). Ouro Preto: Universidade Federal de Ouro Preto; 2003.

5 Prado D. Usando o Arena em simulação. Belo Horizonte: INDG; 2004. vol. 3.

Recebido em: 6 Fev. 2016

Aceito em: 25 Jul. 2016 